\title{
Avaliação neurológica de recém-nascidos pré-termo de muito baixo peso com displasia broncopulmonar
}

\author{
Neurological assessment of very low birth weight infants with bronchopulmonary dysplasia
}

Tathiana Ghisi de Souzaํ, Mônica Sanchez Stopíglia², Emílio Carlos E. Baracat ${ }^{3}$

\section{RESUMO}

Objetivo: Descrever e comparar a avaliação neurológica e comportamental de recém-nascidos pré-termos com e sem displasia broncopulmonar (DBP).

Métodos: Recém-nascidos prematuros com peso ao nascer inferior a $1500 \mathrm{~g}$ e idade gestacional menor de 32 semanas foram avaliados com 40 semanas de idade gestacional corrigida, no Centro de Atenção Integral à Saúde da Mulher da Universidade Estadual de Campinas. Utilizou-se a Avaliação Neurológica de Dubowitz, com 29 itens divididos em seis categorias: tônus, padrões de tônus, reflexos, movimentos, sinais anormais e comportamento. $\mathrm{O}$ estado de consciência do recém-nascido foi graduado segundo Brazelton (1973). Utilizaram-se os testes do qui-quadrado e exato de Fischer para variáveis qualitativas e o de Mann-Whitney para as numéricas não-paramétricas, com nível de significância de $5 \%$.

Resultados: No período de janeiro de 2005 a setembro de 2007, 24 recém-nascidos, 12 com DBP e 12 controles, com idade gestacional ao nascer de $28 \pm 1$ semana e peso de $884 \pm 202 \mathrm{~g}$ no grupo com DBP e $31 \pm 1$ semana e $1156 \pm 216 \mathrm{~g}$ no Grupo Controle foram avaliados. Dos 29 itens avaliados, 18 foram homogêneos entre os grupos e a pontuação geral dos dois grupos não apresentou diferença $(p=0,30)$. Observou-se maior anormalidade neurológica no grupo com DBP em oito itens e, no Grupo Controle, em três itens.

Conclusões: A comparação da avaliação neurológica de Dubowitz de recém-nascidos pré-termos com e sem DBP não apresentou diferença significante com 40 semanas de idade gestacional corrigida. Nas categorias reflexos e postura/tônus, observou-se tendência a anormalidade no grupo DBP.

Palavras-chave: displasia broncopulmonar; prematuro; desenvolvimento infantil.

\section{ABSTRACT}

Objective: To compare the neurological assessment of preterm newborn infants with and without bronchopulmonary dysplasia (BPD).

Methods: Preterm newborn infants with birth weight less than 1,500g and gestational age less than 32 weeks were evaluated by Dubowitz Method at 40 weeks of corrected gestational age. All infants were assisted at Universidade Estadual de Campinas (Unicamp), Campinas (SP), Brazil. Dubowitz Neurological Assessment comprised 29 items divided into six categories: tone, tone patterns, reflexes, movements, abnormal signs and behavior. Newborn behavioral state was graded in six degrees according to Brazelton (1973). Chi-square and Fisher exact tests were used for qualitative variables and Mann-Whitney for non-parametric numeric variables, with $5 \%$ significance level.

Results: From January of 2005 to September of 2007, 24 newborns were evaluated: 12 with BPD (mean gestational age and birth weight of $28 \pm 1$ week and $884 \pm 202 \mathrm{~g}$ ) and 12 without BPD $(31 \pm 1$ week and $1156 \pm 216 \mathrm{~g})$. Out of the 29 items, 18 were homogeneous between the groups. The general score of the groups were similar $(p=0.30)$. For eight items, BPD Group presented higher percentage of abnormalities. Infants without BPD had more abnormalities in three items.
Instituição: Universidade Estadual de Campinas (Unicamp), Campinas, SP, Brasil

${ }^{1}$ Fisioterapeuta, especialista em Fisioterapia Neonatal, Mestre em Saúde da Criança e do Adolescente da Faculdade de Ciências Médicas (FCM) da Unicamp, Campinas, SP, Brasil

${ }^{2}$ Fisioterapeuta, Mestre em Neurociências, responsável pela área de Fisioterapia Neonatal do Centro de Atenção Integral à Saúde da Mulher (Caism) da Unicamp, Campinas, SP, Brasil

${ }^{3}$ Professor-assistente, Doutor do Departamento de Pediatria da FCMUnicamp, Campinas, SP, Brasil
Endereço para correspondência:

Tathiana Ghisi de Souza

Rua Luis Oliveira, 327, apto. 232 - Parque das Flores

CEP:13087-610 - Campinas/SP

E-mail: tathi_neo@yahoo.com.br

Recebido em: 3/6/08

Aprovado em: 20/8/08 
Conclusions: Dubowitz Neurological Assessment in preterm newborn infants with and without BPD did not present significant differences at 40 weeks of corrected gestational age. For reflex and posture/tone categories, there was a trend towards the presence of more abnormalities in the BPD Group.

Key-words: bronchopulmonary dysplasia; infant, premature; child development.

\section{Introdução}

Recém-nascidos prematuros correspondem a 7-10\% de todos os nascimentos, mas concentram aproximadamente $85 \%$ de todas as complicações perinatais ${ }^{(1)}$. A sobrevida de prematuros extremos ( $<28$ semanas de gestação) tem aumentado em decorrência da difusão do uso de surfactante no tratamento da síndrome do desconforto respiratório, juntamente com a administração de glicocorticoides antenatais e novas estratégias de ventilação mecânica. Entretanto, neste grupo de crianças, ainda persiste um alto risco para o desenvolvimento de lesões pulmonares e no sistema nervoso central ${ }^{(1)}$.

A displasia broncopulmonar (DBP) é uma doença cuja etiologia não está totalmente estabelecida, tendo sua origem em múltiplos fatores que afetam o pulmão prematuro. Baixo peso ao nascer, idade gestacional, síndrome do desconforto respiratório e ventilação mecânica são descritos como os fatores de risco mais comuns para o desenvolvimento da doença. Pacientes com DBP geralmente passam por longos períodos de internação e são expostos a vários tratamentos com medicamentos como diuréticos e esteroides pós-natais. Tal terapia vem sendo relacionada a um desfecho neurológico adverso em longo prazo $^{(2)}$.

Diversos fatores podem predispor recém-nascidos prematuros a apresentar déficits cognitivos, sendo a hipóxia crônica, com ou sem isquemia, uma variável significante. $\mathrm{O}$ cérebro do prematuro é exposto à hipóxia por uma série de condições que desencadeiam alterações na relação ventilação-perfusão, resultando também em períodos de hipercapnia e acidose ${ }^{(3)}$. Além disso, recém-nascidos com DBP necessitam de oxigênio suplementar por longos períodos e apresentam episódios recorrentes de bronco-obstrução ${ }^{(3)}$. Outra interferência que pode contribuir para a evolução insatisfatória de crianças com DBP é atribuída à interrupção do desenvolvimento do cérebro, com o parto prematuro. Considerando que, no terceiro trimestre da gestação, o desenvolvimento cerebral é extre- mamente ativo, intervenções médicas, em particular o uso de corticoide, pode ter efeito tóxico direto, com necrose neuronal e interferência em processos de cicatrização ${ }^{(4)}$. Thompson et al demonstraram que prematuros com DBP apresentam uma diminuição global no volume cerebral comparados a prematuros sem a doença ${ }^{(5)}$. Desordens motoras também são descritas em associação à DBP. Alterações específicas, com movimentos mais afetados em braços, pescoço, tronco e na região oro-buco-lingual, são relatadas ${ }^{(6)}$.

A avaliação neurológica de Dubowitz vem sendo usada ao longo das últimas décadas em vários trabalhos envolvendo recém-nascidos prematuros, pois é realizada rapidamente, com instruções simples e sem necessidade de treinamento por parte do examinador ${ }^{(7)}$. Utilizando este instrumento, a identificação antecipada, ainda na fase neonatal, das alterações neurológicas e comportamentais dos recém-nascidos prematuros com DBP pode orientar quanto à adoção de estratégias terapêuticas dirigidas, iniciadas precocemente, e que apresentam potencial para melhorar o prognóstico neurológico global desses pacientes em longo prazo. Assim, o objetivo deste trabalho foi descrever e comparar a avaliação neurológica e comportamental de recém-nascidos pré-termos com e sem DBP.

\section{Métodos}

A amostra do estudo foi composta por uma série de 24 recém-nascidos prematuros, 12 com DBP e 12 controles, sem a doença, com peso ao nascimento inferior a $1500 \mathrm{~g}$ e idade gestacional menor que 32 semanas, nascidos no Centro de Atenção Integral à Saúde da Mulher (Caism) da Unicamp e avaliados pelo método de Dubowitz com 40 semanas de idade gestacional corrigida. A inclusão foi realizada com as admissões consecutivas ocorridas na unidade neonatal, no período de janeiro de 2005 a setembro de 2007. Definiu-se a idade gestacional como a idade em semanas completas de gestação, avaliada conforme os métodos New Ballard ${ }^{(8)}$, Capurro $^{(9)}$, amenorreia e ultrassonografia, em ordem decrescente de preferência.

Excluíram-se os neonatos que, no momento da avaliação, encontravam-se em ventilação mecânica, em uso de medicamentos que interferiram no estado de consciência e em qualquer das seguintes situações clínicas: infecção, sepse, choque, instabilidade hemodinâmica, enterocolite necrosante na época do exame, hemorragia peri-intraventricular Grau III e IV, leucomalácia periventricular, asfixia perinatal e malformações congênitas do sistema nervoso 
central. Para o diagnóstico de lesões do sistema nervoso central, utilizou-se o exame ultrassonográfico cerebral, realizado rotineiramente em todos os recém-nascidos prematuros em três momentos: nas primeiras 72 horas de vida, sete dias após o primeiro exame e com um mês de vida. Todos os recém-nascidos avaliados não apresentavam, no momento da avaliação, processos infecciosos, instabilidade hemodinâmica ou doença concomitante em atividade e foram diagnosticados como estáveis pela equipe médica da unidade neonatal. A asfixia perinatal foi diagnosticada pela presença de pelo menos três dos seguintes critérios: Apgar de quinto minuto menor que seis, tempo de reanimação após ventilação com cânula traqueal maior que um minuto, encefalopatia hipóxico-isquêmica e acometimento sistêmico atribuível à asfixia ${ }^{(10)}$.

A DBP foi diagnosticada nos recém-nascidos que permaneceram dependentes de oxigênio em concentrações acima de $21 \%$ por um período maior ou igual a 28 dias, de acordo com o protocolo de atendimento do Serviço de Neonatologia do Caism da Unicamp, que segue critérios propostos por Jobe e Bancalari ${ }^{(11)}$ e publicados por Monte ${ }^{(12)}$.

Os pacientes foram divididos em dois grupos: com DBP (estudo) e sem DBP (controle). Foi utilizada a Avaliação Neurológica de Dubowitz ${ }^{(13)}$. Tal ferramenta é usada em recém-nascidos prematuros, sendo composta por 34 itens dos quais 29 foram avaliados e divididos em seis categorias: postura e tônus, padrões de tônus, reflexos, movimentos, padrões e sinais anormais, orientação e comportamento. Cinco itens não foram avaliados, três deles (orientação auditiva, orientação visual e alerta) em virtude da interferência do ambiente da terapia intensiva nos resultados, e dois (reflexos tendinosos e reflexo de sucção) por dificuldades técnicas e de material. Todos os recém-nascidos foram avaliados pelo mesmo examinador (autora principal). Os seguintes critérios foram aplicados durante o exame:

- O estado de consciência no momento do exame foi graduado por meio dos seis graus definidos por Brazelton ${ }^{(14)}$, iniciando-se a avaliação entre os estados um e dois e prosseguindo-se nos estados três e quatro.

- As avaliações foram efetuadas entre as mamadas, na Unidade de Terapia Semi-intensiva Neonatal e no Ambulatório de Fisioterapia Neonatal (até uma semana da alta), quando o paciente completava 40 semanas de idade gestacional corrigida.

- O teste de cada item avaliado foi repetido três vezes, sendo registrada a resposta predominante na ficha de avaliação.
Quando o recém-nascido não se encaixava em nenhuma das possibilidades, optava-se pelo diagrama mais próximo.

- Todas as crianças avaliadas se encontravam em boas condições clínicas.

- Os recém-nascidos foram classificados, ao final, em normais, anormais e suspeitos, segundo critérios propostos por Dubowitz ${ }^{(13)}$.

Os dados foram armazenados e analisados a partir do programa SPSS 7.5. Os testes utilizados para a avaliação dos dois grupos foram o qui-quadrado e o exato de Fischer para variáveis qualitativas e o de Mann-Whitney para variáveis numéricas não paramétricas. Foram considerados significantes os resultados que apresentaram um valor de $p$ inferior a $5 \%$.

A pesquisa teve início após a autorização informada dos responsáveis. Este trabalho foi aprovado pelo Comitê de Pesquisa do Caism e pelo Comitê de Ética em Pesquisa da Faculdade de Ciências Médicas da Unicamp.

\section{Resultados}

No período de janeiro de 2005 a setembro de 2007, 62 recém-nascidos preenchiam os critérios do estudo, mas 42 (18 com DBP e 24 controles) tinham um ou mais critérios de exclusão. Dessa forma, 24 recém-nascidos, 12 com DBP com idade gestacional ao nascer de $28 \pm 1$ semana e peso de $884 \pm 202 \mathrm{~g}$ e 12 controles com $31 \pm 1$ semana e $1156 \pm 216 \mathrm{~g}$ foram avaliados.

Das variáveis analisadas, peso e idade gestacional ao nascer, gênero, dias de ventilação mecânica e uso de cateter nasal apresentaram diferença significante entre os grupos: os pacientes com DBP apresentavam peso e IG de nascimento menores e maior permanência em ventilação mecânica e cateter nasal. $\mathrm{O}$ boletim de Apgar de primeiro e quinto minutos não mostrou diferença entre os grupos $(p>0,05)$, conforme Tabela 1.

Predominou o gênero masculino $(n=8)$ no grupo de pacientes com DBP e feminino no grupo sem a doença $(n=8)$. Das doenças perinatais analisadas, a sepse foi mais prevalente no grupo com $\operatorname{DBP}(p=0,01)$. A persistência do canal arterial e o refluxo gastresofágico estiveram presentes em maior porcentagem de neonatos com DBP, mas não houve significância estatística (Tabela 2). Em relação à adequação do peso ao nascimento, oito recém-nascidos foram classificados como pequenos para a idade gestacional (PIG) e quatro adequados para a idade gestacional (AIG), com a mesma distribuição em ambos os grupo. $\mathrm{O}$ uso de corticoide pós-natal foi realizado 
Tabela 1 - Características gerais dos prematuros com e sem displasia broncopulmonar

\begin{tabular}{lccccccccc}
\hline & \multicolumn{3}{c}{ Grupo DBP (n=12) } & \multicolumn{3}{c}{ Grupo Controle (n=12) } & \multirow{p}{c}{ (n) } \\
& Média & Mediana & Mínimo & Máximo & Média & Mediana & Mínimo & Máximo & \\
\hline Peso ao nascer (g) & $884 \pm 202$ & 882 & 575 & 1170 & $1156 \pm 216$ & 1162 & 755 & 1480 & $<0,05$ \\
IG (semanas) & $28 \pm 1$ & 29 & 26 & 31 & $31 \pm 1$ & 31 & 29 & 34 & $<0,05$ \\
VM (dias) & $12 \pm 12$ & 9 & 0 & 47 & $1 \pm 2$ & 0 & 0 & 5 & $<0,05$ \\
Cateter nasal (dias) & $40 \pm 28$ & 36 & 0 & 85 & $4 \pm 10$ & 0 & 0 & 32 & $<0,05$ \\
Apgar 1 & $5 \pm 3$ & 7 & 1 & 9 & $6 \pm 2$ & 7 & 3 & 9 & 0,38 \\
Apgar 5 & $9 \pm 1$ & 10 & 6 & 10 & $9 \pm 1$ & 10 & 7 & 10 & 0,40 \\
\hline
\end{tabular}

VM: ventilação mecânica; IG: idade gestacional ao nascer

Tabela 2 - Prevalência de doenças nos prematuros com e sem displasia broncopulmonar

\begin{tabular}{lccc}
\hline & Grupo DBP $(\mathbf{n}=\mathbf{1 2})$ & Grupo Controle $(\mathbf{n}=\mathbf{1 2})$ & $\boldsymbol{p}$ \\
\hline Sepse & $80 \%$ & $20 \%$ & 0,01 \\
PCA & $77 \%$ & $22 \%$ & 0,08 \\
RGE & $66 \%$ & $33 \%$ & 0,64 \\
DMH & $62 \%$ & $37 \%$ & 0,66 \\
\hline
\end{tabular}

PCA: persistência do canal arterial; RGE: refluxo gastresofágico; DMH: doença de membrana hialina.

em todos os recém-nascidos com DBP e em nenhum recémnascido do Grupo Controle.

As respostas dos recém-nascidos aos itens avaliados no exame neurológico não apresentaram diferenças entre os grupos. Dos 29 itens avaliados, nas seis categorias, 18 itens foram classificados como homogêneos entre os grupos $(p=1,00)$ e a pontuação geral dos dois grupos foi similar $(p=0,30)$. Nos itens colocação plantar, elevação da cabeça em prono e irritabilidade, os neonatos do Grupo Controle apresentaram maior percentual de anormalidade (Tabela 3).

Quando os dados foram analisados de maneira descritiva, observou-se um percentual maior de anormalidade no grupo com DBP em oito dos 29 itens da avaliação neurológica: tração do braço, recuo da perna, tração da perna, controle de cabeça 2 (avaliação do tônus flexor cervical), tônus flexor 2 (comparação entre tônus flexor braço versus perna), tônus extensor aumentado, preensão palmar e preensão plantar. Nos itens tônus extensor cervical, posturas anormais de pés e mãos e susto, os pacientes do Grupo Controle apresentaram maior percentual de anormalidade (Tabela 4).

\section{Discussão}

Comparar os achados neurológicos de crianças com DBP com dados disponíveis na literatura é difícil, tanto pela escassez de trabalhos sobre o assunto, quanto pelo método adotado $^{(15)}$. Sabe-se que, quanto menor a idade gestacional, maior a chance de se desenvolver $\mathrm{DBP}^{(2)}$. Em nosso estudo, o grupo com DBP apresentou idade gestacional e peso de nascimento significativamente menores do que aqueles apresentados no Grupo Controle.

Apesar de a etiologia da DBP ainda ser controversa, alguns fatores de risco devem ser ressaltados. Neste estudo, as variáveis peso e idade gestacional ao nascer, dias de ventilação mecânica e de cateter nasal apresentaram diferenças com os controles. Além disso, a incidência de doenças como sepse, persistência do canal arterial e refluxo gastresofágico foi maior no grupo com DBP. Tais achados são concordantes com os de Tapia et al, que identificaram, como principais fatores de risco para o desenvolvimento de DBP, o baixo peso ao nascer, a idade gestacional mais jovem, a presença de síndrome do desconforto respiratório, o uso de ventilação mecânica, a infecção neonatal e a persistência do canal arterial ${ }^{(2)}$.

A influência da DBP no neurodesenvolvimento de recém-nascidos de muito baixo peso tem gerado controvérsias. Nossos achados coincidem com os de Byrne et al, que estudaram o desenvolvimento motor em idade corrigida a termo de recém-nascidos de muito baixo peso a partir da Avaliação Neurológica de Dubowitz ${ }^{(16)}$, não encontrando diferença entre os portadores e não portadores de DBP. No presente trabalho também não existiu diferença significante entre os grupos, porém a porcentagem de anormalidade nos 
Tabela 3 - Anormalidade nos grupos de recém-nascidos pré-termos com e sem displasia broncopulmonar nos itens considerados homogêneos na avaliação neurológica de Dubowitz

\begin{tabular}{|c|c|c|c|c|}
\hline & Variável & $\begin{array}{c}\text { Grupo DBP } \\
\quad(n=12)\end{array}$ & $\begin{array}{l}\text { Grupo Controle } \\
\qquad(n=12)\end{array}$ & $p$ \\
\hline \multirow[t]{6}{*}{ Postura e tônus } & Postura & $75 \%$ & $56 \%$ & NS \\
\hline & Recuo do braço & $25 \%$ & $25 \%$ & NS \\
\hline & Ângulo poplíteo & $16 \%$ & $8 \%$ & NS \\
\hline & Controle de cabeça ${ }^{1}$ & $16 \%$ & $8 \%$ & NS \\
\hline & Resposta à tração & $25 \%$ & $25 \%$ & NS \\
\hline & Suspensão ventral & $75 \%$ & $66 \%$ & NS \\
\hline \multirow[t]{2}{*}{ Padrões de tônus } & Tônus flexor ${ }^{1}$ & zero & zero & - \\
\hline & Tônus extensor da perna & $25 \%$ & $25 \%$ & NS \\
\hline \multirow[t]{2}{*}{ Reflexos } & Colocação plantar & zero & $8 \%$ & NS \\
\hline & Reflexo de moro & $41 \%$ & $41 \%$ & NS \\
\hline \multirow[t]{3}{*}{ Movimentos } & Espontâneos (qualitativo) & $8 \%$ & zero & NS \\
\hline & Espontâneos (quantitativo) & $8 \%$ & zero & NS \\
\hline & $\begin{array}{l}\text { Elevação da cabeça em } \\
\text { prono }\end{array}$ & $8 \%$ & $16 \%$ & NS \\
\hline Padrões e sinais anormais & Tremor & $25 \%$ & $25 \%$ & NS \\
\hline \multirow[t]{4}{*}{ Orientação e comportamento } & Aparência dos olhos & $25 \%$ & $16 \%$ & NS \\
\hline & Irritabilidade & $16 \%$ & $41 \%$ & NS \\
\hline & Consolabilidade & $33 \%$ & $25 \%$ & NS \\
\hline & Choro & $75 \%$ & $66 \%$ & NS \\
\hline
\end{tabular}

NS: não significante.

Tabela 4 - Comparação do percentual de anormalidade entre os grupos de recém-nascidos pré-termos com e sem displasia broncopulmonar, nos itens considerados não homogêneos na avaliação neurológica de Dubowitz

\begin{tabular}{llccc}
\hline & Variável & $\begin{array}{c}\text { Grupo DBP } \\
(\mathbf{n = 1 2})\end{array}$ & $\begin{array}{c}\text { Grupo Controle } \\
(\mathbf{n = 1 2})\end{array}$ & $\boldsymbol{p}$ \\
\hline Postura e tônus & Tração do braço & $67 \%$ & $42 \%$ & NS \\
& Recuo da perna & $83 \%$ & $58 \%$ & NS \\
& Tração da perna & $42 \%$ & $17 \%$ & NS \\
& Controle de cabeça ${ }^{2}$ & $33 \%$ & $17 \%$ & NS \\
Padrões de tônus & Tônus flexor ${ }^{2}$ & $25 \%$ & $8 \%$ & NS \\
& Tônus extensor cervical & $25 \%$ & $42 \%$ & NS \\
Reflexos & Tônus extensor aumentado & $17 \%$ & zero & zS \\
Padrões e sinais anormais & Posturas anormais dos & $17 \%$ & zero & NS \\
& Preensão palmar & $25 \%$ & $50 \%$ & \\
& Preensão plantar & $42 \%$ & & \\
\end{tabular}

NS: não significante. 
itens avaliados foi maior no grupo com DBP, com exceção das variáveis colocação plantar, elevação da cabeça em prono, posturas anormais de pés e mãos, susto e irritabilidade, para as quais os pacientes do Grupo Controle apresentaram maior percentual de anormalidade.

Perlman e Volpe ${ }^{(6)}$ descreveram uma desordem de movimento em bebês com DBP semelhante à Coreia, com envolvimento de membros superiores e inferiores. Esse achado é raro no primeiro ano de vida de recém-nascidos saudáveis e, mesmo em crianças com desordens de movimento causadas por lesões nos gânglios basais, tal anormalidade apresentase, geralmente, após o primeiro ano. No referido estudo, os autores relataram tais movimentos anormais nos primeiros meses de idade de prematuros com $\mathrm{DBP}^{(6)}$. Na presente investigação, não verificou-se esse tipo de anormalidade de movimento; apenas tremores estiveram presentes em $25 \%$ dos recém-nascidos de ambos os grupos. Talvez, no momento da avaliação (40 semanas), tais alterações potenciais estivessem ausentes, o que não exclui a possibilidade de seu achado alguns meses após o nascimento.

A doença pulmonar crônica tem um efeito deletério no desenvolvimento neuromotor precoce, mesmo na ausência de lesões estruturais, como a hemorragia e a leucomalácia periventricular. Na comparação entre prematuros com e sem DBP, há diferenças no desempenho de certas funções motoras, perceptivas e de inteligência ${ }^{(17)}$. Em estudo feito por Mercuri et a ${ }^{(18)}$, comparou-se a capacidade dos recém-nascidos prematuros e a termo de manter diferenças entre os padrões de tônus (flexor e extensor), entre a região cervical, membros superiores e inferiores. A adoção de um padrão flexor em determinado momento e extensor em outro é, provavelmente, o mais importante sinal de integridade neurológica. Em nosso estudo, a maioria dos recém-nascidos com e sem DBP apresentou uma distribuição normal de tônus, porém o grupo com DBP apresentou o dobro de recém-nascidos com padrão anormal, sem capacidade de adotar alternância entre flexão e extensão, mantendo uma postura de braços fletidos e pernas estendidas.

A melhor resposta dos recém-nascidos com DBP ao item elevação da cabeça em prono pode ser explicada pela permanência desses bebês por longos períodos em decúbito ventral, protocolo de posicionamento preferencial para crianças com doenças pulmonares e doença do refluxo gastresofágico. Esse posicionamento estimula o fortalecimento dos músculos extensores do pescoço, facilitando o movimento de elevação da cabeça em prono. Em um estudo comparativo feito por Mercuri et al, é citada a possível influência da adoção desse tipo de posicionamento no encontro de tônus extensor cervical predominante em recém-nascidos pré-termo ${ }^{(18)}$.

A utilização do posicionamento terapêutico é recente. Monterosso et $a l^{(19)}$, em 2003, ressaltam a importância da adoção do posicionamento terapêutico em unidade de terapia intensiva neonatal, já que a restrição da mobilidade associada à postura prona ou supina pode resultar em alterações transitórias do tônus muscular, afetando até recém-nascidos prematuros sem doenças neurológicas. No presente estudo, os bebês avaliados foram posicionados de acordo com o protocolo de posicionamento terapêutico realizado no hospital, com variações de decúbito ao longo do dia.

As variáveis comportamentais, susto e irritabilidade, tiveram maior incidência de anormalidades nos recémnascidos sem DBP. Tais achados poderiam ser justificados pela manipulação frequente de prematuros com DBP no ambiente hospitalar, que potencialmente levaria à tolerância às intervenções médicas e de enfermagem ${ }^{(20)}$. Este ponto é controverso ao se considerar que o maior tempo de permanência em terapia intensiva poderia levar à desorganização neurológica do pré-termo e, portanto, à presença mais frequente de alterações comportamentais. Outra justificativa seria a necessidade de reserva de energia, pois recém-nascidos com DBP apresentam dificuldade de alimentação, com quedas constantes de saturação de oxigênio durante a mamada, dificuldade de coordenação entre sucção, deglutição e respiração e gasto energético maior para manter tais funções ${ }^{(21)}$.

Alterações no desenvolvimento de recém-nascidos prematuros com DBP têm sido cada vez mais descritas na literatura, havendo relação com a gravidade da DBP e o maior risco de sequelas no neurodesenvolvimento. Entretanto, a maioria dos trabalhos aborda crianças em idade escolar ou pré-escolar, como os recentes estudos de Short et al e de O'Shea et al ${ }^{(22,23)}$. Além dos efeitos diretos no desenvolvimento neurocomportamental durante o período neonatal, um efeito adicional deletério da DBP poderia ser atribuído às reinternações frequentes durante o primeiro ano de vida. Em estudo publicado por $\mathrm{Greenough}^{(24)}$, observou-se que aproximadamente $50 \%$ das crianças com DBP são readmitidas em hospitais durante a infância por desconforto respiratório e, ainda, que a taxa de readmissão é maior nas crianças com infecções provocadas pelo vírus sincicial respiratório. Os agravos repetidos, as internações frequentes, o ambiente hospitalar e fatores presentes em idades fora do período neonatal podem ter impacto adicional negativo no desenvolvimento neurológico, além das condições presentes até 40 semanas de vida ${ }^{(24)}$. 
Os resultados deste estudo, que não revelou diferenças significantes no exame neurológico com 40 semanas de idade gestacional corrigida em recém-nascidos com e sem DBP, também demonstrou tendência de anormalidade no grupo com DBP nas categorias reflexos e postura/tônus. O pequeno número de recém-nascidos estudados foi uma limitação da pesquisa e essa tendência para achados anormais no exame

\section{Referências bibliográficas}

1. Thébaud B, Lacaze-Masmonteil T, Watterberg K. Postnatal glucocorticoids in very preterm infants: "the good, the bad, and the ugly"? Pediatrics 2001;107:413-5.

2. Tapia JL, Agost D, Alegria A, Standen J, Escobar M, Grandi C; NEOCOSUR Collaborative Group. Bronchopulmonary dysplasia: incidence, risk factors and resource utilization in a population of South American very low birth weight infants. J Pediatr (Rio J) 2006;82:15-20.

3. Raman L, Georgieff MK, Rao R. The role of chronic hypoxia in the development of neurocognitive abnormalities in preterm infants with bronchopulmonary dysplasia. Dev Sci 2006;9:359-67.

4. Anderson PJ, Doyle LW. Neurodevelopmental outcome of bronchopulmonary dysplasia. Semin Perinatol 2006;30:227-32.

5. Thompson DK, Warfield SK, Carlin JB, Pavlovic M, Wang HX, Bear M et al. Perinatal risk factors altering regional brain structure in the preterm infant. Brain 2007;130:667-77.

6. Perlman JM, Volpe JJ. Movement disorder of premature infants with severe bronchopulmonary dysplasia: a new syndrome. Pediatrics 1989;84:215-8.

7. Dubowitz L, Ricciw D, Mercuri E. The Dubowitz neurological examination of the full-term newborn. Ment Retard Dev Disabil Res Rev 2005;11:52-60.

8. Ballard JL, Khoury JC, Wedig K, Wang L, Eilers-Walsman BL. New Ballard score, expanded to include extremely premature infants. J Pediatr 1991;119:417-23.

9. Capurro H, Konichezky S, Fonseca D, Calobeyro-Borcia R. A simplified method for diagnosis of gestacional age in the newborn infant. J Pediatr 1978;93:120-2.

10. Rosa IR, Marba ST. Fatores de risco para asfixia neonatal em recém nascidos com peso acima de 1000 gramas. J Pediatr (Rio J) 1999;75:50-4.

11. Jobe AH, Bancalari E. Bronchopulmonary dysplasia. Am J Respir Crit Care Med 2001;163:1723-9.

12. Monte LF, Silva Filho LV, Miyoshi MH, Rozov T. Bronchopulmonary dysplasia. J Pediatr (Rio J) 2005;81:99-110

13. Dubowitz LM, Dubowitz V, Mercuri E. The neurological assessment of the preterm \& full term newborn infant. London: Cambridge; 1999. neurológico de recém-nascidos pré-termos com DBP poderia ser comprovada com uma amostra maior de pacientes. Outra sugestão é o acompanhamento longitudinal desses pacientes, particularmente nos primeiros anos de vida, para definir a participação real dos agravos e intercorrências clínicas no neurodesenvolvimento, com a utilização de outros instrumentos de avaliação neurocomportamental e cognitiva.

14. Brazelton TB. Neonatal behavioral assessment scale. London: William Heinemann Medical Books; 1973.

15. Amador JC, Condino-Neto A. Growth and development in infants with bronchopulmonary dysplasia: a prospective study. Rev Cienc Med Campinas 2004;13:23-31.

16. Byrne PJ, Piper MC, Darrah J. Motor development at term of very low birthweight infants with bronchopulmonary dysplasia. J Perinatol 1989;9:301-6.

17. Katz-Salamon M, Gerner EM, Jonsson B, Lagercrantz H. Early motor and mental development in very preterm infants with chronic lung disease. Arch Dis Child Fetal Neonatal Ed 2000;83:F1-6.

18. Mercuri E, Guzzetta A, Laroche S, Ricci D, Vanhaastert I, Simpson A et al. Neurologic examination of preterm infants at term age: comparison with term infants. J Pediatr 2003;142:647-55.

19. Monterosso L, Kristjanson LJ, Cole J, Evans SF. Effect of postural supports on neuromotor function in very preterm infants to term equivalent age. J Paediatr Child Health 2003;39:197-205.

20. Brown NC, Doyle LW, Bear MJ, Inder TE. Alterations in neurobehavior at term reflect differing perinatal exposures in very preterm infants. Pediatrics 2006;118:2461-71.

21. Mizuno K, Nishida Y, Taki M, Hibino S, Murase M, Sakurai M et al. Infants with bronchopulmonary dysplasia suckle with weak pressures to maintain breathing during feeding. Pediatrics 2007;120:e1035-42.

22. Short EJ, Kirchner HL, Asaad GR, Fulton SE, Lewis BA, Klein N et al. Developmental sequelae in preterm infants having a diagnosis of bronchopulmonary dysplasia: analysis using a severity-based classification system. Arch Pediatr Adolesc Med 2007;161:1082-7.

23. O'Shea TM, Nageswaran S, Hiatt DC, Legault C, Moore ML, Naughton M et al. Follow-up care for infants with chronic lung disease: a randomized comparison of community- and center-based models. Pediatrics 2007;119:e947-57.

24. Greenough A. Bronchopulmonary dysplasia - long term follow up. Paediatr Respir Rev 2006;7(Suppl 1):S189-91. 